\section{Keratinocyte stem cells: targets for cutaneous carcinogens}

\author{
Rebecca J. Morris
}

Lankenau Institute for Medical Research, 100 Lancaster Avenue west of City Line, Wynnewood,

Pennsylvania 19096, USA. Phone: (610) 645-8475; Fax: (610) 645-2205; E-mail: Ricercar@tigger.jvnc.net.

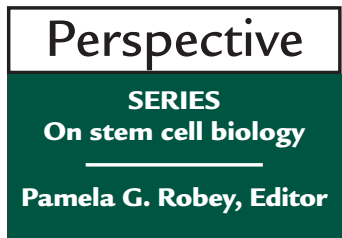

A skin cancer seen in the clinic is in reality the culmination of a long history, only the later stages of which are easily observed. Progression from normal growth to the neoplastic, and later to the metastatic, stage of disease may involve multiple changes in cellular phenotypes and patterns of gene expression. Nonetheless, the early events in the pathway that leads from exposure to mutagenic agents to the formation of skin papillomas or carcinomas can be viewed as a two-step process of tumor initiation and tumor promotion. Fundamental questions remain about this process. In particular, the identity and the biological properties have yet to be defined of those cells, existing within a larger population of basal keratinocytes, that are specifically subject to tumor initiation. In addition, the behavior of these cells before, during, and after tumor promotion remains uncertain. The rare cells in this tissue that are capable of becoming pre-neoplastic when treated with a carcinogen exhibit many of the properties expected of keratinocyte stem cells. Here, I consider the evidence that they are indeed stem cells, and I explore the implications of this hypothesis for carcinogenesis in the skin.

Based on the hierarchical stem cell model of cellular replacement - originally described by Gilbert and Lajtha $(1,2)$ for the hematopoietic bone marrow, and later discussed by Buick (3) and by Hume (4) - a stem cell represents a self-renewing cell that may also produce proliferative cells, which undergo a series of amplification divisions prior to terminal maturation. Stem cells are thought to be quiescent or to cycle slowly under normal circumstances and to be protected by the architecture of the tissue. They should also be capable, when appropriately stimulated, of extensive proliferation to produce terminally differentiated progeny, and they should be capable of self-renewing in vivo and in vitro in order to maintain their own population as well as that of the terminally differentiated cells. Furthermore, some but not all stem cells are multipotential, that is, capable of producing more than one terminally differentiated lineage. Given this definition, do the targets of carcinogen action meet this definition of a stem cell (5)?

Keratinocyte stem cells have not yet been isolated, but some of their features are now becoming evident. From work in my laboratory and others, it is now possible to identify subpopulations with varying proliferative potential within the basal layer of the epidermis. This, along with other characteristics, suggests that some of these cells correspond to true stem cells. Keratinocyte subpopulations can be defined by various characteristics - location within the tissue, responses to chemical treatments, mitotic behavior in vivo or in vitro, morphology, and expression of marker proteins. Each of these parameters helps define a subpopulation that may contain stem cells, but none of them, taken individually, provides an accepted operational definition of the keratinocyte stem cell. Together, however, these parameters show great promise for the identification and isolation of keratinocyte stem cells. I will focus on the properties of these candidate stem cell populations and the possible identities among them, as well as on the evidence that these cells are the targets of two-stage carcinogenesis.

\section{Tumor initiation and promotion}

Cutaneous neoplasms are not observed after a single (subtumorigenic) exposure of mouse skin to a chemical carcinogen. However, this treatment suffices to initiate tumors, which develop within weeks of treatment with a second, tumor-promoting agent, which induces chronic regenerative hyperplasia. Tumor initiation is thought to convert some of the epidermal keratinocytes into latent neoplastic cells, but the epidermis is visibly unchanged and continues to turn over as usual. Promotion, with an agent such as $12-O-$ tetradecanoylphorbol-13-acetate (TPA), elicits expression of the neoplastic change, but only in tissue that was previously exposed to mutagenic chemicals or irradiation (for review, see ref. 6). Effective initiators of skin carcinogenesis, such as benzo $[a]$ pyrene $(\mathrm{B}[\mathrm{a}] \mathrm{P})$ and 7,12-dimethylbenz[a] anthracene (DMBA), bind covalently to the DNA and thereby permanently alter the genome of epidermal keratinocytes. Interestingly, whether promotion is begun 1 week or 1 year after the exposure to the carcinogen, the tumor responses evoked are surprisingly similar, suggesting that the latent initiated cells neither proliferate nor are lost over long periods. The persistence of these initiated cells in the epidermis, apparently for the life of the animal, is remarkable in view of the normal continuous turnover of cells in the epidermis and the cyclic growth and regression of hair follicles

Although agents such as DMBA are incorporated into, and act in, the nuclei of all epidermal cells, only a minority of these can contribute to the reservoir of cells, then, represent the target of two-stage carcinogenesis. In principle, target cells would be expected to be among those normal cells that avoid the constant turnover seen in most of the epidermis, and they should also possess a high intrinsic capacity for proliferation. These clues point to the epidermal stem cell as the likely target for carcinogens. latent neoplastic cells after tumor initiation. These rare 


\section{Heterogeneity among keratinocytes}

The epidermis is a continually renewing tissue, normally balancing cellular proliferation in the basal layer with loss from the suprabasal layer through a process of terminal differentiation (for reviews, see refs. 5, 7). The basal layer in adult mice consists primarily of keratinocytes, although Langerhans cells (representing about $10 \%$ of the cells in this layer) and melanocytes (about 3\%) can also be found, as can a small number of special sensory cells, Merkel cells. Hair follicles (shown in Figure 1) are specialized cutaneous appendages, contiguous with both the interfollicular epidermis and the sebaceous glands.

Although at first glance the basal epidermal cells of mice appear microscopically quite uniform, more careful investigation of whole mounts of epidermal sheets and vertical cross sections through the dorsal epidermis of the mouse shows that this tissue is organized into morphologically defined proliferative units (Figure 2). Well prepared vertical cross sections reveal a single layer of basal cells covered with a suprabasal layer consisting of regularly spaced columns of three flattened nucleated cells and occasional cells between the columns (Figure 2a). Epidermal proliferative units, most easily visualized in silver-stained epidermal whole mounts, contain groups of 10-12 basal cells positioned in a spiral pattern within the margins of the flattened suprabasal cells (Figure 2b). Two or three of the basal cells in each group are close to the central column of suprabasal nuclei. The remaining, peripheral basal cells in each proliferative unit are usually more than one nuclear diameter from the center.

The mitotic activities of these central and peripheral cells are quite distinct. Epidermal DNA synthesis and mitosis vary in a circadian manner, with the morning hours marking the peak of mitotic activity, and the evening hours marking the peak of DNA synthesis. Following a single injection of $\left[{ }^{3} \mathrm{H}\right]$ thymidine in the morning hours, about $5 \%$ of the nuclei in peripheral cells take up the label and are visible in light microscopic autoradiographs. Nuclei in the central cells do not become labeled after this brief exposure. Mitotic figures are also easy to find among the peripheral nuclei but are rarely seen in the central cells, indicating that most of the cell division among basal keratinocytes occurs in the periphery of these proliferative units. Although occasional cells with pulse-labeled nuclei remain on the basal layer for a month or more, these cells typically remain on the basal layer for only 4 to 5 days before they are displaced to the suprabasal layers and are found in the columns. Hence, these pulselabeled cells behave as though they were more mature or more differentiated than the central basal cells.

Although nuclei of central cells are not readily labeled by a short pulse of $\left[{ }^{3} \mathrm{H}\right]$ thymidine, they can be labeled by exposure to $\left[{ }^{3} \mathrm{H}\right]$ thymidine for a week or more, suggesting that they cycle far more slowly than peripheral cells. This suggestion was borne out by the work of Bickenbach and Mackenzie $(8,9)$, and shortly thereafter by Potten (10) and this laboratory (11). Different labeling methods and different ages of mice were used, but with similar results: When the entire basal layer is labeled by continuous administration of $\left[{ }^{3} \mathrm{H}\right]$ thymidine, central cells retained the label for 1 to 6 months longer than the peripheral cells. Bickenbach et al. demonstrated that the reduction in grain count over these "label-retaining" cells was consistent with a very long cell cycle for this population; label-retaining cells do indeed proliferate in vivo, albeit at a very slow rate (12).

Label-retaining epidermal cells have also been observed in the middle third of the hair follicles immediately beneath the sebaceous gland (13). Silver et al. also reported retention of label in the melanocytes of the hair follicle germ (14), but the precise distribution of the highly persistent, label-retaining cells within the epithelial, mesenchymal, and pigment cellular compartments of the hair follicle needs to be addressed in greater detail.

\section{The epidermal hierarchy and cutaneous carcinogenesis}

Experiments with TPA provide the first line of evidence that label-retaining, but not pulse-labeled, basal keratinocytes can contribute to the reservoir of pre-neoplastic cells (9). In normal nonmutagenized mouse skin, basal cell nuclei can be labeled nearly uniformly by a long series of injections with $\left[{ }^{3} \mathrm{H}\right]$ thymidine. When these cells are allowed to mature for a month, so that maturing cells exit the basal layer, a brief treatment with TPA drives most of the label-retaining cells (which remain located in the central region of the proliferative units) into mitosis. Conversely, after brief exposure to $\left[{ }^{3} \mathrm{H}\right]$ thymidine to preferentially label the peripheral, maturing cells, pulse-labeled cells respond to TPA by exiting the cell cycle and moving to the suprabasal layers of the skin, where differentiated keratinocytes reside. These observations suggest that the two classes of epidermal basal cells differ not only with respect to their positions in the tissue architecture and relative time spent on the basal layer, but also with respect to their early responses to TPA: Label-retaining cells proliferate, whereas most pulse-labeled cells differentiate.

Also consistent with a specific role for label-retaining basal keratinocytes in two-stage carcinogenesis is the finding that these cells retain carcinogen molecules

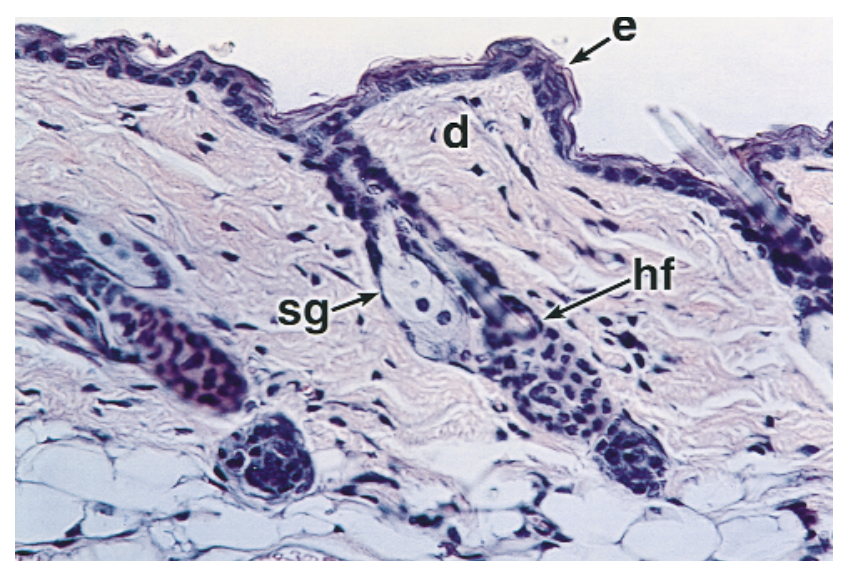

\section{Figure 1}

Cross section of the epidermis demonstrating the epidermis (e) overlaying the dermis (d), and a hair follicle (hf) together with its sebaceous gland (sg). Original magnification, $\times 400$. 
Table 1

Characteristics of label-retaining and maturing basal keratinocytes

\begin{tabular}{|c|c|c|}
\hline & Label-retaining basal cells & Mature basal cells \\
\hline \multirow[t]{5}{*}{ In vivo } & Identified by long term $[3 \mathrm{H}]$ thymidine & Identified following a single pulse of [3H]thymidine \\
\hline & Located in the central portion of the & \\
\hline & proliferative unit and in the permanent region of the hair follicle & Located on the periphery of the proliferative unit \\
\hline & Proliferate in response to TPA & Do not proliferate in response to TPA \\
\hline & Retain carcinogens & \\
\hline \multirow[t]{3}{*}{ In vitro } & Included in the clonogenic epidermal population (kCFC) & Are rarely clonogenic \\
\hline & Differentiate in response to TPA & Do not survive treatment with TPA \\
\hline & Small, dense cells, high ratio of nuclear volume to cytoplasmic volume & Not as small and dense as label-retaining cells \\
\hline
\end{tabular}

adducted to their DNA (15). Radiolabeled B $[a]$ P can be used in place of $\left[{ }^{3} \mathrm{H}\right]$ thymidine to label cells over the entire thickness of the skin. Shortly after this treatment, labeling is greatest over the hair follicles, sebaceous glands, and interfollicular epidermis, but over the succeeding weeks, most of the material is lost from the basal layer, consistent with the overall pattern of epidermal renewal. After a month, only about $2 \%$ of interfollicular basal cells retain the carcinogen, but these cells appear identical to the label-retaining cells described above: They cycle slowly, reside in the central regions of the epidermal proliferative units, and respond rapidly to TPA by undergoing mitosis. The identity of these cell populations has been confirmed by double isotope-double emulsion autoradiography, using $\left[{ }^{3} \mathrm{H}\right]$ thymidine and $\left[{ }^{14} \mathrm{C}\right] \mathrm{B}[\mathrm{a}] \mathrm{P}$ to test for the presence and location of doubly labeled cells.

These observations suggest that the slowly cycling subpopulation of epidermal cells participates in the initiation phase of carcinogenesis, not necessarily because they retain the carcinogen adducts, but because they remain in the basal cell layer, rather than undergo terminal differentiation like the remainder of the basal cells. Carcinogen-retaining cells can also be found at low levels in hair follicles. As shown in Figure 3, these cells reside in the middle, permanent region of the hair follicle, termed the "bulge," so called because the cells in this region are subject neither to the more rapid turnover of the inter- and intrafollicular epidermis, nor to the cyclic growth and regression of the lower region of the hair follicle.

\section{Behavior of the basal keratinocyte subpopulations in vitro}

To explore the properties of label-retaining and maturing basal cells in culture, we have prepared keratinocytes labeled in vivo with the familiar procedures of prolonged or brief exposure to $\left[{ }^{3} \mathrm{H}\right]$ thymidine, and we have cultured these cells on plates of irradiated feeder cell layers for extended periods. By following the distribution of radiolabeled nuclei, we were able to compare the fates of the two subpopulations in vitro (16). After 5 days, label-retaining cells were found as pairs and clusters having silver grain counts, but pulse-labeled epidermal cells remained primarily as single cells. Hence, labelretaining cells divide to form colonies (albeit small ones), and they appear to possess greater proliferative potential than do pulse-labeled cells, which form colonies only rarely. The cells that divide to form colonies in this assay, defined as keratinocyte CFCs $(\mathrm{kCFCs})$, may therefore represent the in vitro correlate of keratinocyte stem cells, which retain label and persist in the epidermis.

Preparations of basal keratinocytes may be separated on the basis of size and density, using Percoll gradient sedimentation. This procedure may be of great value, since it can be used to enrich for immature basal cells and avoids the complications that could arise from incorporating radioactivity into the cells (17). No clear separation is possible with this approach, since pulselabeled cells are found throughout the gradient, but the densest fraction, which is morphologically characterized by cells with a high nucleus-to-cytoplasm ratio, is enriched for slowly cycling, label-retaining (or carcinogen-retaining) cells, while maturing cells are enriched in lighter fractions.

Although TPA treatment in vivo appears to drive the majority of pulse-labeled basal keratinocytes to differentiate, this treatment applied to cultured primary keratinocytes causes massive cell death. However, within preparations of normal epidermal cells, some basal cells remain attached and apparently unscathed by the TPA. Upon continued treatment, these cells are capable of long-term proliferation and proceed to stratify as do untreated cultures. Since this characteristic might be expected of a fairly immature population of epidermal basal cells, we have characterized this population of TPA-resistant cells using density gradient sedimentation (17). TPA treatment, begun 24 hours after plating, caused cells from all fractions to detach and to die, but many more attached cells remained from the densest cell fraction than from the lighter fractions. Thus, Percoll density gradients can be used to enrich for basal cells that are either inherently resistant to TPA, or that respond to it primarily by proliferation. Somehow, these cells and their progeny must be able to adapt to the TPA, because the TPA-treated cultures eventually stratify and are practically indistinguishable morphologically from the control cultures. Whether TPA-resistant cells are identical to label-retaining cells in vivo is not yet clear, but the fraction of relatively dense cells is indeed enriched, 

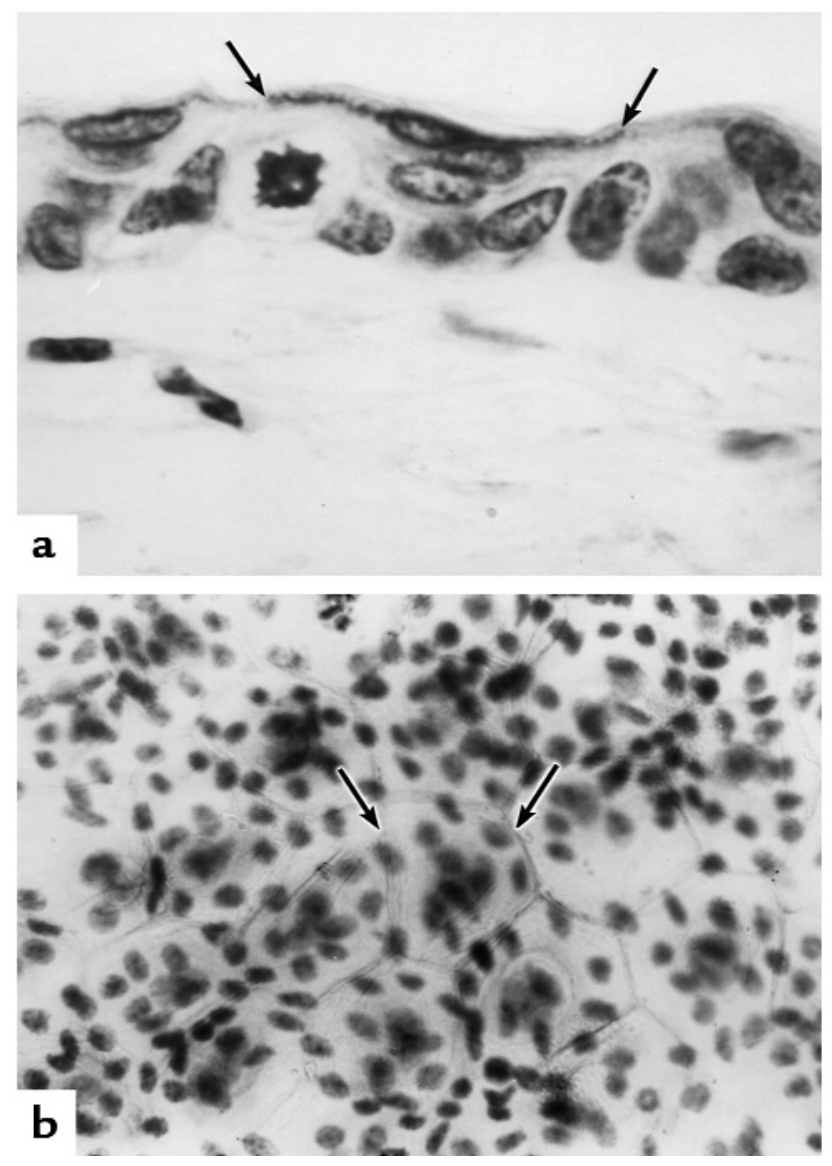

Figure 2

The epidermal proliferative unit, shown in cross section (a) and in an epidermal whole mount (b). In a, note the columnar arrangement of the differentiating suprabasal cells. The edges of the outermost suprabasal cell (arrows) correspond to the silver-stained boundaries shown below. Note the mitotic basal cell beneath the edge of the suprabasal column. Original magnification, $\times 1000$. In $\mathbf{b}$, a surface view of the dorsal epidermis of the mouse is shown, demonstrating the hexagonal arrangement of the platelike cells in the stratum corneum (arrows) following silver impregnation. The nuclei of underlying basal cells are stained with hematoxylin. Original magnification, $\times 630$.

about twofold, for cells that can form colonies in vitro. Since such kCFCs appear to include labelretaining cells, this finding suggests that the populations defined by these different parameters include a similar set of relatively immature basal keratinocytes. Some of the distinguishing properties of the basal cell subpopulations are shown in Table 1.

Recent results indicate that fluorescence-activated cell sorting (FACS) or even differences in adhesive properties may be used to enrich for keratinocyte stem cells. Jones et al. (18) have demonstrated that keratinocytes having characteristics of stem cells (such as the ability to form colonies and to reconstitute a graft) may be selected by their relatively high level of $\beta 1$ integrin subunit expression. Bickenbach and Chism (19) also used the expression of $\beta 1$ integrin subunit to enrich for labelretaining keratinocytes based upon their greater adherence than more differentiated cells to type IV collagen. Similarly, Li et al. (20) used the expression of the inte- grin subunit $\alpha 6$, as well as the lack of expression of the epitope of a monoclonal antibody, 10G7, to enrich for keratinocyte stem cells. The $\alpha 6$ integrin subunit is known to be expressed on all basal keratinocytes, whereas the monoclonal antibody $10 \mathrm{G} 7$ is expressed on actively proliferating cells but not on quiescent cells. By FACS analysis, cells having very high total proliferative output, as expected for stem cells, were $\alpha 6^{+} / 10 G 7^{-}$. This approach could be used with functional studies, as described above, to provide a greater enrichment for the target cells in epidermal carcinogenesis.

\section{kCFCs in two-stage carcinogenesis}

The kCFC assay provides a well recognized, quantifiable indicator of the number of cells with relatively high growth potential and of the relative growth potential of single cells (21). From normal, untreated mice 9 to 59 weeks of age, the number of kCFCs remains essentially unchanged during adult life, with an average colony-forming efficiency of $0.45 \%$. To determine the effects of in vivo initiation or promotion on the number of in vitro clonogenic epidermal cells, we treated 8-week-old mice topically with DMBA and assayed kCFCs from their skin either with or without subsequent TPA treatment. At all times tested, for more than a year after initiation, DMBA-treated skin contained a number of kCFCs within the untreated control range. TPA treatment alone caused an increase in the number of kCFCs over untreated control skin, but interestingly, TPA treatment of initiated epidermis caused a still greater increase in kCFCs. The stable number of kCFCs argues against the possibility that initiated cells undergo expansion. In keeping with earlier evidence that responses to TPA are similar independent of time after initiation, we can thus confirm that the number of keratinocytes initiated by carcinogen-treatment remains constant.

Direct evidence that keratinocytes in the cutaneous epithelium can persist for such long intervals comes from our observations made at 14 months after complete labeling of the epidermis and hair follicles of neonatal mice with $\left[{ }^{3} \mathrm{H}\right]$ thymidine $(22)$. Three-dimensional reconstruction of dorsal underfur follicles from serial sections from these mice disclosed the presence of highly persistent label-retaining cells associated with the first-generation follicle involved in the production of the first wave of hairs, commonly called the bulge. These highly persistent label-retaining cells were most often found on the ventral surface of the first-generation follicle, five cell positions from the base, near the site of attachment of the arrector pilorum muscle. No highly persistent label-retaining cells were found in the hair canal, sebaceous gland, hair germ, or interfollicular epidermis after the 14-month interval.

To determine whether the target cells in two-stage carcinogenesis are not only persistent but also quiescent, we took advantage of the ability of 5-fluorouracil (5-FU) to kill cycling cells specifically (23). We first established that topically applied 5-FU could cause a transient loss of epidermal proliferation: Within a day, this treatment causes a $40 \%$ reduction in the number of basal cells in the interfollicular epi- 
dermis, and it virtually abolishes DNA synthesis, an effect that lasts 2 days. With basal cell maturation blocked for at least 7 days, the epidermis is reduced and, in many cases, sloughs off completely. Hair growth is also blocked but resumes within about 12 days. By applying 5-FU either before or after tumor initiation, we established that this drug acts neither as an initiator nor as a promoter of carcinogenesis. Furthermore, the similar tumor latency, the similar tumor yields, and the similar final number of papillomas and carcinomas under both conditions suggest that the cells initiated by the DMBA are resistant to the effects of the 5-FU.

We conclude from these studies that the epithelial cells initiated by the carcinogen are quiescent, rather than actively cycling. If so, it may be that neoplastic lesions are not fixed in these cells until tumor promotion is begun, at which time the target cells are induced to undergo multiple rounds of DNA synthesis. This suggestion is consistent with the observation that carcinogens remain adducted to DNA in central basal cell nuclei of slowly cycling, label-retaining cells. Carcinogen-DNA interactions that date from the initial exposure to mutagenic agents are retained until the time of tumor promotion, at which point the damage and, hence, the transforming mutation become fixed within the genome.

Where, then, do these quiescent initiated cells reside? Argyris (24) suggests a follicular origin for papillomas and carcinomas, based on a model of epidermal abrasion that was found to be a tumor-promoting stimulus. The Argyris abrasion protocol entails removing the interfollicular epidermis but leaves the hair follicles undisturbed. Our studies with 5-FU support his conclusion, with the additional finding that the follicular infundibulum can be excluded as a target region, because it becomes damaged and atrophic following administration of 5-FU. Furthermore, the hair germ can also be excluded, because the mice were treated in anagen, the stage in the hair growth cycle when the germ cells are most sensitive to the killing action of the 5 -FU. Consequently, the middle, permanent region of the follicle beneath the sebaceous gland is the most likely location of the initiated cells.

To follow up on this possibility, we used the Argyris method to remove the interfollicular epidermis of carcinogen-initiated mice, and we compared their tumor responses to those of initiated but unabraded mice. The interfollicular epidermis of the abraded group of mice quickly regenerated from cells in the hair follicles, after which time, tumor promotion with TPA was begun in both groups. Mice in which the interfollicular epidermis had been previously removed developed papillomas and carcinomas (25), and carcinoma responses were not significantly different in the abraded and unabraded groups. However, after 40 weeks, papillomas were half as numerous in abraded mice. Hence, the fact that the carcinoma responses were the same in abraded and unabraded mice suggests that stem cells having the greatest proliferative potential are found in the hair follicles. Furthermore, these results support the hypothesis that the target cells for carci-

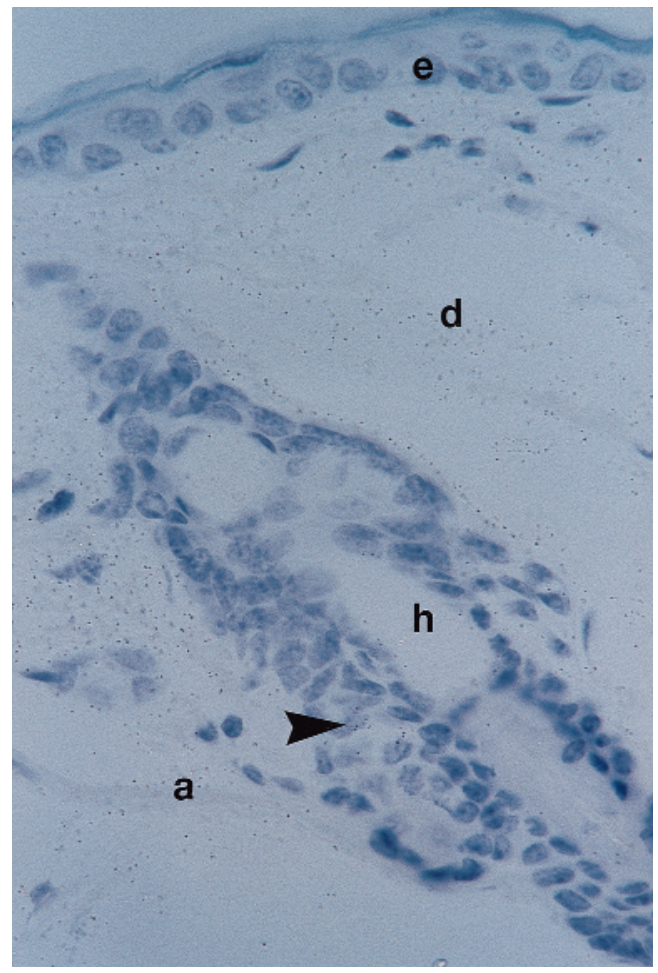

\section{Figure 3}

Carcinogen label-retaining cells in the hair follicle. Eight-week-old mice were treated with a single topical application of $\left[{ }^{3} \mathrm{H}\right] \mathrm{DMBA}$. Seven weeks later, samples of the dorsal skin were fixed, and cross sections were prepared. Note the carcinogen-retaining cell in the middle third of the follicle (arrowhead). e, epidermis; d, dermis; h, hair follicle; a, arrector pilorum muscle. Original magnification, $\times 630$.

nomas and for many papillomas indeed reside in the hair follicles, although the target cells for some papillomas are also present in the interfollicular epidermis.

\section{Conclusions and prospects}

In view of their continual renewal and persistence in the epidermis, initiated cells may not be simply any proliferative cells, but stem cells. While keratinocyte stem cells have not yet been definitively identified or isolated, results from several laboratories have made great strides toward these goals. Because of their persistence, the recently described label-retaining keratinocytes from the hair follicles may be considered potential targets for two-stage carcinogenesis. In addition, since target cells should show a relatively high intrinsic potential for proliferation, kCFCs, which give rise to colonies, represent another candidate target population. Finally, our experiments with 5-FU indicate that the principal site of the target cells is the middle, permanent region of the hair follicles, although the abrasion studies show that a significant population capable of developing into papillomas, but not carcinomas, exists in the interfollicular epidermis (or infundibulum of the hair follicles).

There are two new and potentially fruitful approaches for the identification and isolation of epidermal targets for chemical carcinogens. The first, mentioned previously, is FACS, which should be especially helpful as new determinants become available for either stem 
cells or maturing proliferating cells. In particular, the sorted subpopulations or the colonies derived from them could be analyzed for the presence of the mutated Ha-ras gene, which is the hallmark of DMBA initiation (for review, see ref. 6), or for mutations in as-yet unidentified genes that affect colony formation or selfrenewal. Another tool of great promise in identifying the target cells is laser capture microdissection (LCM) followed by genetic analysis of the captured cells. LCM enables us to select small foci of cells from hematoxylin and eosin-stained sections of the earliest hyperplastic foci, as well as foci within initiated hair follicles. This approach should lead to the identification of additional molecular determinants for phenotypically distinct subpopulations of keratinocytes, such as stem cells. As these approaches are pursued, we shall no doubt find that the stem cell compartment in the cutaneous epithelium is as complicated and elegant as that of the hemopoietic system.

\section{Acknowledgments}

This work was supported by grant CA-45293-13 from the National Institutes of Health and by the Lankenau Foundation. I thank Tom O'Brien from the Lankenau Institute for Medical Research and George Cotsarelis from the Department of Dermatology of the University of Pennsylvania for critically reading the manuscript and for their suggestions. Because of the nature of this article, I could not give the original citations from the work of my many colleagues, and I ask the reader's understanding.

1. Gilbert, C.W., and Lajtha, L.G. 1965. The importance of cell population kinetics in determining the response to irradiation of normal and malignant tissue. In Cellular radiation biology. Wiliams and Wilkins. Baltimore, Maryland, USA. 118-154

2. Lajtha, L.G. 1979. Stem cell concepts. Differentiation. 14:23-24.

3. Buick, R.N. 1984. Perspectives on clonogenic tumor cells, stem cells, and oncogenes. Cancer Res. 44:4909-4918.

4. Hume, W.J. 1985. Keratinocyte proliferative hierarchies confer protective mechanisms in surface epithelia. Br. J. Dermatol. 112:493-502.

5. Morris, R.J. 1993. Epidermal stem cells: targets for carcinogenic chemicals. Semin. Dev. Biol. 4:251-259.

6. DiGiovanni, J. 1994. Multistage skin carcinogenesis in mice. In Carcino- genesis. M.P. Waalks and J.M. Ward, editors. Raven Press. New York, New York, USA. 265-299.

7. Miller, S.J., Lavker, R.M., and Sun, T.-T. 1997. Keratinocyte stem cells of cornea, skin, and hair follicles. In Stem cells. C.S. Potten, editor. Academic Press. San Diego, California, USA. 331-362.

8. Bickenbach, J.R. 1981. Dentification and behavior of label-retaining cells in oral mucosa and skin. J. Dent. Res. 60:1611-1620.

9. Mackenzie, I.C., and Bickenbach, J.R. 1985. Localization of label-retaining cells in mouse epithelia. Cell Tissue Res. 242:551-556.

10. Potten, C.S. 1986. Cell cycles in cell hierarchies. Int. J. Radiat. Biol. 49:257-258.

11. Morris, R.J., Fischer, S.M., and Slaga, T.J. 1985. Evidence that the centrally and peripherally located cells in the murine epidermal proliferative unit are two distinct cell populations. J. Invest. Dermatol. 34:277-281.

12. Bickenbach, J.R., McCutecheon, J., and Mackenzie, I.C. 1986. Rate of loss of tritiated thymidine label in basal cells in mouse epithelial tissues. Cell Tissue Kinet. 19:325-333.

13. Cotsarelis, G., Sun, T.-T., and Lavker, R.M. 1990. Label-retaining cells reside in the bulge area of pilosebaceous unit: implications for follicular stem cells, hair cycle, and skin carcinogenesis. Cell. 61:1329-1337.

14. Silver, A.F., Chase, H.B., and Potten, C.S. 1969. Melanocyte precursor cells in the hair follicle germ during dormant stage (telogen). Experientia. 25:299-301.

15. Morris, R.J., Fischer, S.M., and Slaga, T.J. 1986. Evidence that a slowly cycling subpopulation of adult murine epidermal cells retains carcinogen. Cancer Res. 46:3061-3066.

16. Morris, R.J., and Potten, C.S. 1994. Slowly cycling (label-retaining) epidermal cells behave like clonogenic stem cells in vitro. Cell Prolif. 27:279-289.

17. Morris, R.J., Fischer, S.M., Klein-Szanto, A.J.P., and Slaga, T.J. 1991. Subpopulations of primary adult murine epidermal basal cells sedimented on density gradients. Cell Tissue Kinet. 23:587-602.

18. Jones, P.H., Harper, S., and Watt, F.M. 1995. Stem cell patterning and fate in human epidermis. Cell. 80:83-93.

19. Bickenbach, J.R., and Chism, E. 1998. Selection and extended growth of murine epidermal stem cells in culture. Exp. Cell Res. 244:184-195.

20. Li, A., Simmons, P.J., and Kaur, P. 1998. Identification and isolation of candidate human keratinocyte stem cells based on surface phenotype. Proc. Natl. Acad. Sci. USA. 95:3902-3907.

21. Morris, R.J., Tacker, K.C., Fischer, S.M., and Slaga, T.J. 1988. Quantitation of primary in vitro clonogenic keratinocytes from normal adult murine epidermis, following initiation, and during promotion of epidermal tumors. Cancer Res. 48:6285-6290.

22. Morris, R.J., and Potten, C.S. 1999. Highly persistent label-retaining cells in the hair follicles of mice and their fate following induction of anagen. J. Invest. Dermatol. 112:470-475.

23. Morris, R.J., Coulter, K., Tryson, K., and Steinberg, S.R. 1997. Evidence that cutaneous carcinogen-initiated epithelial cells from mice are quiescent rather than actively cycling. Cancer Res. 57:3435-3443.

24. Argyris, T.S. 1985. Promotion of epidermal carcinogenesis by repeated damage to mouse skin. Am. J. Ind. Med. 8:329-337.

25. Morris, R.J., Tryson, K., and Wu, K.Q. 2000. Evidence that epidermal targets of carcinogen action are found in the interfollicular epidermis or infundibulum as well as in the hair follicles. Cancer Res. 60:226-229. 